\title{
ASSISTED VENOUS DRAINAGE PRESENTS THE RISK OF UNDETECTED AIR MICROEMBOLISM
}

Angelo LaPietra, MD

Eugene A. Grossi, MD

Bradley B. Pua, BS

Rick A. Esposito, MD

Aubrey C. Galloway, MD

Christopher C. Derivaux, MD

Lawrence R. Glassman, MD

Alfred T. Culliford, MD

Greg H. Ribakove, MD

Stephen B. Colvin, MD
Objectives: The proliferation of minimally invasive cardiac surgery has increased dependence on augmented venous return techniques for cardiopulmonary bypass. Such augmented techniques have the potential to introduce venous air emboli, which can pass to the patient. We examined the potential for the transmission of air emboli with different augmented venous return techniques.

Methods: In vitro bypass systems with augmented venous drainage were created with either kinetically augmented or vacuum-augmented venous return. Roller or centrifugal pumps were used for arterial perfusion in combination with a hollow fiber oxygenator and a $40-\mu \mathrm{m}$ arterial filter. Air was introduced into the venous line via an open 25-gauge needle. Test conditions involved varying the amount of negative venous pressure, the augmented venous return technique, and the arterial pump type. Measurements were recorded at the following sites: pre-arterial pump, post-arterial pump, postoxygenator, and patient side.

Results: Kinetically augmented venous return quickly filled the centrifugal venous pump with macrobubbles requiring continuous manual clearing; a steady state to test for air embolism could not be achieved. Vacuumaugmented venous return handled the air leakage satisfactorily and microbubbles per minute were measured. Higher vacuum pressures resulted in delivery of significantly more microbubbles to the "patient" $(P<.001)$. The use of an arterial centrifugal pump was associated with fewer microbubbles $(P=.02)$.

Conclusions: Some augmented venous return configurations permit a significant quantity of microbubbles to reach the patient despite filtration. A centrifugal pump has air-handling disadvantages when used for kinetic venous drainage, but when used as an arterial pump in combination with vacuumassisted venous drainage it aids in clearing air emboli. (J Thorac Cardiovasc Surg 2000;120:856-63)
From the Department of Surgery, Division of Cardiothoracic Surgery, New York University School of Medicine, New York, NY.

Read at the Eightieth Annual Meeting of The American Association for Thoracic Surgery, Toronto, Ontario, Canada, April 30-May 3, 2000.

Received for publication April 24, 2000; revisions requested May 26, 2000; revisions received June 1, 2000; accepted for publication July 12, 2000.

Address for reprints: Eugene A. Grossi, MD, New York University Medical Center, Suite 9-V, 530 First Ave, New York, NY 10028 (E-mail: grossi@cv.med.nyu.edu).

Copyright (C) 2000 by The American Association for Thoracic Surgery

$0022-5223 / 2000 \$ 12.00+0 \quad \mathbf{1 2 / 6 / 1 1 0 1 8 3}$

doi:10.1067/mtc.2000.110183 nduction of venous blood return to the venous reservoir during cardiac surgery is typically initiated by a gravity siphon and maintained by the height of the patient with respect to the venous reservoir. With the introduction of minimally invasive techniques, however, peripheral cannulation and smaller venous cannulas have increased the resistance to venous blood flow and have necessitated the use of augmented venous return techniques. These augmented drainage systems can be either vacuum-augmented venous return or kinetically augmented venous return. Both of these systems create a large negative pressure in the venous line, either through vacuum assistance or by a pump added to the 


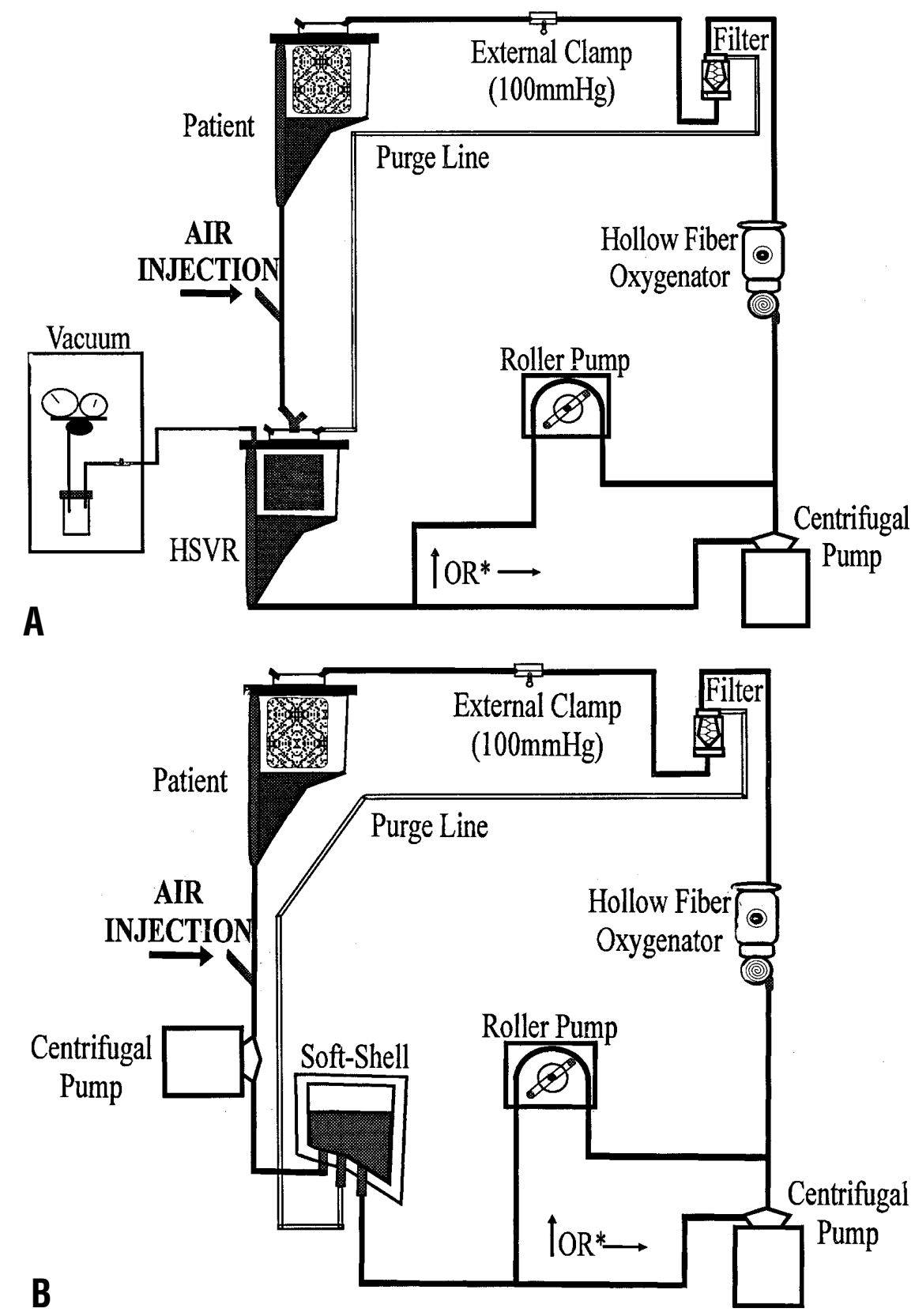

Fig 1. A, Vacuum-augmented venous return CPB circuit. *Arterial pump was alternated between roller and centrifugal for each part of the experiment. $H S V R$, Affinity hard shell venous reservoir. B, Kinetically augmented venous return CPB circuit. *Arterial pump was alternated between roller and centrifugal for each part of the experiment. Soft-Shell, Soft shell venous reservoir.

venous circuit. Creation of a subatmospheric pressure increases the venous pressure gradient between the patient and the venous inlet of the pump circuit and allows satisfactory flow to be maintained through smaller, high-resistance cannulas. As a result of these augmented systems, venous return can be increased without the need to raise the patient's height with respect to the venous reservoir.

Recently it has been shown that augmented venous return techniques may help to introduce gaseous microemboli into the patient undergoing cardiopulmonary bypass (CPB). ${ }^{1}$ Although many potential caus- 


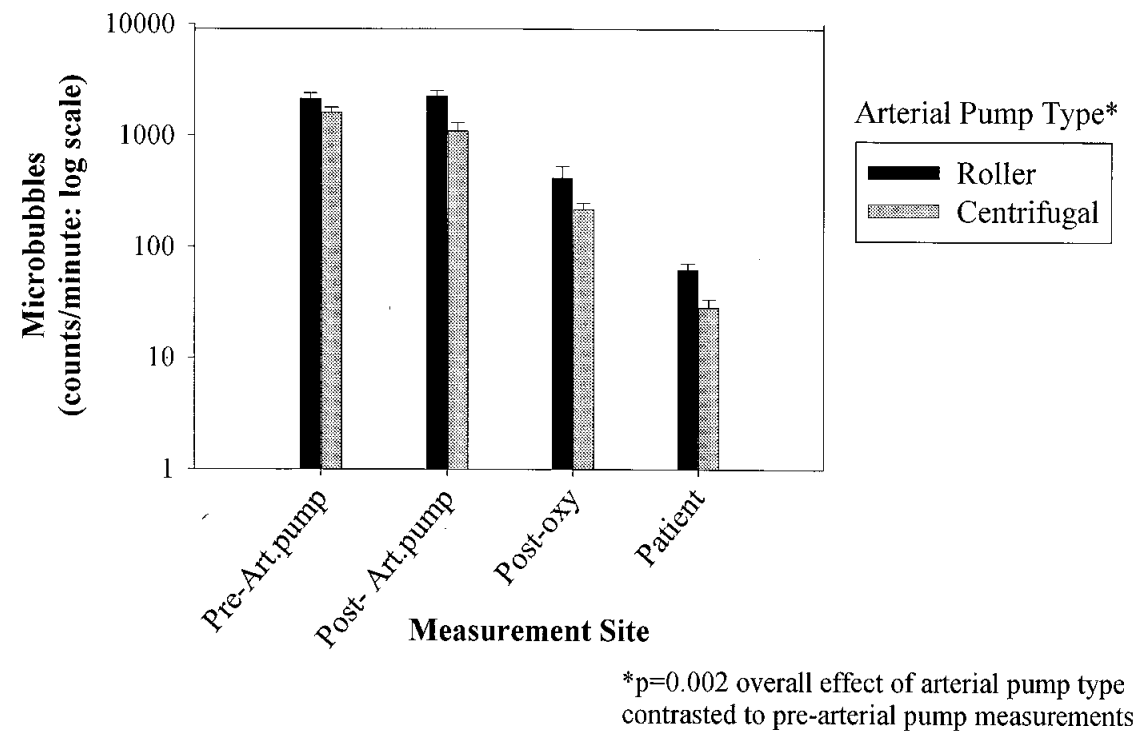

Fig 2. Total microbubbles at different CPB circuit sites for all vacuum levels. Pre-Art., Pre-arterial, Post-Art., postarterial; Post-oxy, post-oxygenator. *Statistically significant overall effect of arterial pump type.

Table I. Known causes of gaseous microemboli within a $C P B$ circuit

\author{
Bubble oxygenator \\ Damaged membrane material \\ Counter-diffusion \\ Overoccluded roller pump \\ Excessive cardiotomy/vent suction \\ Inadequate debubbling \\ Cavitation \\ Low reservoir levels \\ Excessive temperature gradients \\ Injections into the circuit \\ Mechanical manipulations of the circuit
}

Modified from Kurusz M, Butler BD. Embolic events and cardiopulmonary bypass (page 274). In: Gravlee GP, Davis RP, Utley JR, editors. Cardiopulmonary bypass: principles and practice. Baltimore: Williams \& Wilkins; 1993:267-290

es for gaseous emboli during CPB have been identified (Table I),$^{2}$ the creation of a negative pressure in the venous line facilitates entrapment of air around the venous cannula, possibly increasing gaseous microemboli. This is of concern, since emboli, whether blood, foreign material, or gaseous, are believed to be responsible for the neurocognitive impairment that some patients experience after $\mathrm{CPB} .^{2}$

In this experimental series, we compared the potential for gaseous microembolus formation and transmission with the use of augmented venous return systems. Concomitantly, we evaluated the gaseous microembolus handling capabilities of various clinical augmented venous return $\mathrm{CPB}$ circuits.

\section{Materials and methods}

Four in vitro experimental $\mathrm{CPB}$ circuits were constructed with $13-\mathrm{mm}(3 / 8-i n c h)$ diameter tubing. All of the individual circuits were constructed of new components consisting of the following basic elements: (1) a patient simulating reservoir (HSVR shell, Affinity NT 541; Medtronic, Inc, Minneapolis, Minn), (2) an augmented venous return system, (3) an arterial pump, (4) a hollow fiber membrane oxygenator (Affinity NT 541; Medtronic), (5) a 40- $\mu \mathrm{m}$ arterial line filter (model M40; Medtronic), and (6) an arterial line that led back to the patient reservoir (outer shell of HSVR, Affinity NT 541; Medtronic). The arterial pump was either a roller pump (Sarns, Inc, Ann Arbor, Mich) or a centrifugal pump (BP-80; Medtronic). The augmented venous return systems consisted of either vacuum or kinetic assistance. In the vacuum-augmented venous return system, a hard-shell venous reservoir (Affinity; Medtronic) was connected to a vacuum regulator system, which controlled the amount of negative pressure applied to the system (Fig 1, A). In the kinetically augmented venous return system, a centrifugal pump (BP-80) was incorporated into the venous line between the venous cannula and a soft-shell venous reservoir (MVR 1600; Medtronic) (Fig 1, B).

After the circuits had been flushed with carbon dioxide, ${ }^{1,3}$ Ringer's solution was used as a prime. The circuits were at room temperature and no gas flow was placed through the membrane oxygenator. Test conditions included steady-state flows of $5 \mathrm{~L} / \mathrm{min}$ with a pinch clamp on the arterial line before the "patient." The clamp produced a 100-mm Hg gradient (mimicking the gradient seen across an arterial cannula). The arterial filter was oriented properly and its purge line was left open. A venous line pinch clamp was used to regulate the return from the "patient" and to maintain the venous 

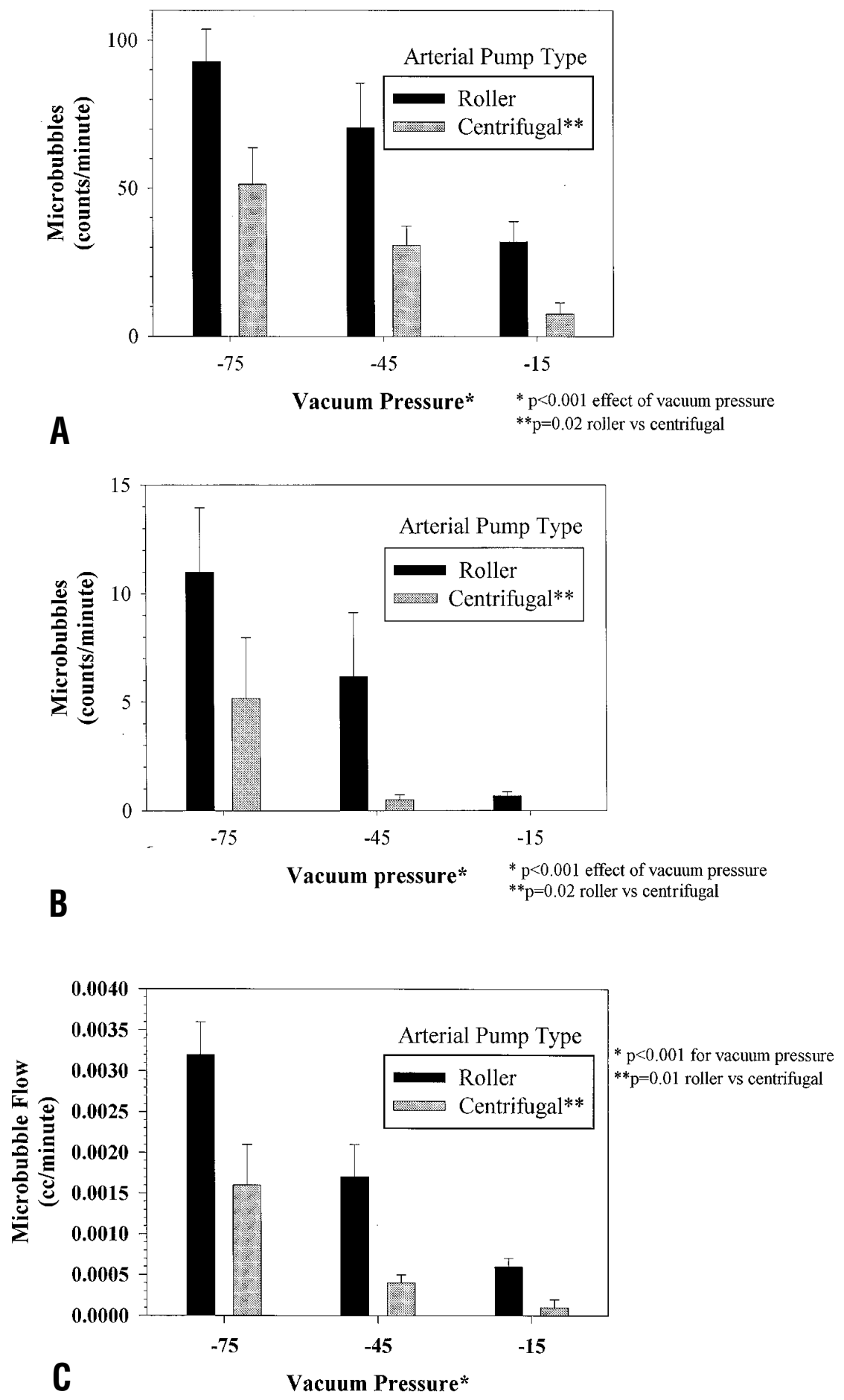

Fig 3. A, Effect of arterial pump type and vacuum pressure on microbubbles $50 \mu \mathrm{m}$ or less in diameter after arterial cannula. *Statistically significant overall effect of vacuum pressure. ${ }^{* *}$ Statistically significant overall effect of arterial pump type. B, Effect of arterial pump type and vacuum pressure on microbubbles 50 to $100 \mu \mathrm{m}$ in diameter after arterial cannulation. *Statistically significant overall effect of vacuum pressure. ${ }^{* *}$ Statistically significant overall effect of arterial pump type. C, Effect of arterial pump type and vacuum pressure on microbubble flow after arterial cannula. ${ }^{*}$ Statistically significant overall effect of vacuum pressure. ${ }^{* *}$ Statistically significant overall effect of arterial pump type. 
Table II. Total microbubbles (mean counts per minute $\pm S D$ ) with a 25-gauge needle in the venous line and vacuum-augmented venous return

\begin{tabular}{lccc}
\hline & & Arterial roller pump* & $-75 \mathrm{~mm} \mathrm{Hg}{ }^{*}$ \\
\cline { 2 - 4 } Measure site & $-15 \mathrm{~mm} \mathrm{Hg \dagger}$ & $-45 \mathrm{~mm} \mathrm{Hg} \boldsymbol{H}^{*}$ & $3081.4 \pm 2104.6(2759.5)$ \\
PAP\$ & $1933.4 \pm 1838.7(1716.0)$ & $1301.4 \pm 729.5(1009.5)$ & $2317.1 \pm 1571.7(2111.5)$ \\
PoAP $\$$ & $1983.4 \pm 1891.4(1767.0)$ & $1187.6 \pm 747.5(1166.5)$ & $1334.0 \pm 121.2(1368.0)$ \\
PO & $89.0 \pm 20.8(85.0)$ & $159.3 \pm 81.3(145.5)$ & $103.8 \pm 23.7(95.5)$ \\
PS & $32.5 \pm 16.7(28.5)$ & $76.7 \pm 41.8(76.5)$ & \\
\hline
\end{tabular}

$S D$, Standard deviation; $P A P$, pre-arterial pump; PoAP, post-arterial pump; $P O$, post-oxygenator; $P S$, patient side. Figures in parentheses indicate medians.

*Main effect of arterial pump type (contrasted to pre-arterial pump values) $\mathrm{F}=9.51 ; P=.002$.

$\dagger$ Covariate effect of negative pressure $\mathrm{F}=6.20 ; P=.01$.

‡Counts per minute made with less sensitive settings due to high number and size of counts.

reservoir level at approximately 300 to $400 \mathrm{~mL}$ while the "patient" shell was maintained at a level of 800 to $1000 \mathrm{~mL}$.

After baseline measurements had been established with varying degrees of venous assistance $(-15 \mathrm{~mm} \mathrm{Hg},-45 \mathrm{~mm}$ $\mathrm{Hg}$, and $-75 \mathrm{~mm} \mathrm{Hg}$ ), air was allowed into the venous line by simply inserting an open-ended 25-gauge hypodermic needle into the venous tubing. The 25 -gauge needle was chosen for its dramatic demonstration of gaseous macroemboli and microembolus entrapment with augmented venous return. This corresponds to a small tear from a suture in the atrial cannulation site, which allows air entry. Air emboli were recorded in various parts of the circuit by the use of a snap-on ultrasound probe with the aid of a coupling gel and a CMD-10 ultrasonic detector (Hatteland Instrumentering, Norway). The size and number of microbubbles, ranging from 20 to $300 \mu \mathrm{m}$ in diameter, could thereby be quantified. ${ }^{4,5}$ The CMD-10 detector, with the aid of a computer program, BUBMON Software (Hatteland Instrumentering, Norway), applied a deconvolution filter to the raw data and generated histograms of bubble size. Microbubbles were quantified at 5 different locations in the CPB circuit (venous inlet, pre-arterial pump, pre-oxygenator, pre-filter, and patient side) for each experimental condition. When bubbles were visually detected, quantification was impossible due to the bubble size limitation of the detector system. At the pre-arterial and post-arterial pump measurement sites, the bubble counter required a lower sensitivity due to the large number and size of bubbles. The post-oxygenator and "patient" sites were recorded on the identical maximum sensitivity scale to allow for direct comparison and evaluation of bubbles as small as $20 \mu \mathrm{m}$ in diameter.

The number and size of microbubbles were measured for 60 -second intervals, and these measurements were repeated a minimum of 6 times for each experimental condition at the specified location. The method of venous drainage was randomized for each circuit. The volumes of bubbles were corrected for line pressures and were reported for 1 atmosphere of pressure. The statistical software package SPSS (SPSS, Inc, Chicago, Ill) was used to analyze the data. Analysis of variance and general linear models modules (SPSS) were used to test for differences due to the test conditions. The Bonferroni method was used for subgroup comparisons. Data are reported as mean \pm standard error.

\section{Results}

In the first part of the experiment, we evaluated the gaseous microembolus handling abilities of the kinetically augmented venous return circuit. With the continuous entrainment of air in the venous line, the venous centrifugal pump and reservoir continuously filled with both macrobubbles and microbubbles and required constant manual clearing. This prevented achievement of steady-state conditions and did not permit appropriate measurements with the CMD-10 ultrasonic detector.

The second experimental series was performed with vacuum-augmented venous return. This pump setup was able to clear the macrobubbles, and steady-state conditions were achieved, that is, no macrobubbles were visualized ahead of the arterial pump. With the steady-state flow of $5 \mathrm{~L} / \mathrm{min}$, the line pressures in the CPB circuit were $215 \mathrm{~mm} \mathrm{Hg}$ after the arterial pump, $113 \mathrm{~mm} \mathrm{Hg}$ after the oxygenator, and $100 \mathrm{~mm} \mathrm{Hg}$ after the $37-\mu \mathrm{m}$ filter.

The data measurements obtained at the preset locations and vacuum pressures of the various vacuum-augmented venous return circuits are presented in Table II. With either arterial pump, an increased negative vacuum pressure was associated with an increase in the number of bubbles per minute seen at the different measuring points (covariate effect of negative pressure $\mathrm{F}$ value $=6.20 ; P=.01$ ). For the measurements prearterial and post-arterial pump, the great number and size of microbubbles necessitated measurement on a less sensitive scale, which undercounts smaller sized bubbles. However, the recordings made post-oxygenator and in the "patient" were done at the maximum sensitivity of the bubble counter, eliminating the possibility of this type of underestimation. Fig 2 graphically compares the handling of gaseous microemboli according to the arterial pump type. The centrifugal arterial pump was associated with lower counts per minute at the sites beyond the pump (overall effect of arterial 


\begin{tabular}{ccc}
\hline & Arterial centrifugal pump* \\
\cline { 2 - 3 }$-15 \mathrm{~mm} \mathrm{H} \dagger$ & $-45 \mathrm{~mm} \mathrm{Hg}{ }^{*}$ & $-75 \mathrm{~mm} \mathrm{Hg \dagger}$ \\
\hline $859.5 \pm 511.1(665.5)$ & $1245.6 \pm 508.5(1037.0)$ & $2188.7 \pm 1683.2(1285.5)$ \\
$273.8 \pm 114.8(257.0)$ & $443.3 \pm 126.1(426.0)$ & $2530.6 \pm 1433.0(2259.5)$ \\
$56.5 \pm 14.7(55.0)$ & $249.8 \pm 48.1(242.0)$ & $439.8 \pm 114.5(411.0)$ \\
$7.5 \pm 9.3(5.5)$ & $31.2 \pm 16.2(26.0)$ & $56.5 \pm 36.4(42.0)$ \\
\hline
\end{tabular}

pump contrasted to pre-arterial pump values, $\mathrm{F}=9.51$; $P=.002)$. Both the oxygenator $(P<.001)$ and arterial filter $(P<.001)$ removed significant gaseous microemboli from the circuit.

These test circuits revealed a mean of $45.4 \pm 35.1$ microbubbles per minute reaching the "patient," estimated at a mean covariate of $-45-\mathrm{mm} \mathrm{Hg}$ vacuum. The histograms in Fig 3, $A$ and $B$, depict the numbers and sizes of bubbles reaching the patient. The mean counts increased with higher negative vacuum pressures $(P<$ $.001)$ and diminished with use of an arterial centrifugal pump ( $P=.02)$. Fig 3, $A$, depicts the distribution of 20to $50-\mu \mathrm{m}$ bubbles, whereas Fig $3, B$, depicts the distribution of 50- to $100-\mu \mathrm{m}$ bubbles. No bubbles larger than $100 \mu \mathrm{m}$ were seen to reach the patient. Fig 3, $C$, shows the flow of microbubbles (mean $0.0013 \pm 0.0002$ $\mathrm{mL} / \mathrm{min}$ ) reaching the patient. Significant increases were associated with increasing negative vacuum pressure $(P<.001)$ and use of an arterial roller pump $(P=.01)$.

\section{Discussion}

Air emboli can enter a patient's venous or arterial circulation during almost any type of surgical procedure. ${ }^{6}$ Detection of these gaseous microemboli is extremely important in patients undergoing cardiac surgery with CPB because these emboli have been implicated as major factors in the postoperative neuropsychologic deficits experienced by some of these patients. ${ }^{7}$ Air originating from the CPB circuit has been found to contribute to the formation of gaseous microemboli. ${ }^{8-11}$

Modifications of the CPB circuit to accommodate minimally invasive cardiac surgical procedures have increased the need for the operative team to understand the formation and handling of gaseous microemboli by the different components of the CPB circuit. ${ }^{1,12,13}$ These modifications include the addition of augmented venous return to compensate for the increased flow resistance in the smaller cannulas being used. The two major types of augmented venous return techniques used clinically include kinetic and vacuum-assisted drainage (Fig 1). Importantly, in this study both of these techniques resulted in significant amounts of air introduction when a hole was present in the venous circuit. In the first experimental series (kinetic technique), the venous centrifugal pump was unable to handle the delivered volume load of continuous gaseous microemboli due to a small continuous air leak, requiring constant manual clearing of the pump and soft reservoir. Certainly this would not be ideal for routine clinical practice.

In the next experimental series, vacuum-augmented venous return configurations satisfactorily handled the macroscopic air entrainment. Despite the presence of a standard $40-\mu \mathrm{m}$ arterial filter, however, gaseous microemboli were consistently delivered to the patient in the setting of a small "air leak" before the venous inlet. This delivery of gaseous microemboli was increased by higher negative vacuum pressures and diminished with the use of a centrifugal pump in the arterial position as compared with a roller pump. Of note is the fact that gaseous microemboli larger than $50 \mu \mathrm{m}$ consistently were delivered to the patient with the combination of higher vacuum and arterial roller pump use. Although the absolute clinical relevance is unknown, this combination should be avoided if possible.

Similarly, Willcox, Mitchell, and Gorman ${ }^{1}$ recently examined the effect of vacuum-augmented venous return on venous air entrained in the CPB circuit, demonstrating a 10-fold increase in gaseous microemboli with the addition of constant vacuum assist (-60 $\mathrm{mm} \mathrm{Hg}$ ). However, this study had several methodologic limitations. A dedicated microbubble counter was not used but, rather, a general-purpose 2-MHz pulsed wave color Doppler ultrasonogram. This device report- 
ed an "index" of bubble counts and was unable to differentiate among the sizes of bubbles. Without a deconvolution filter, undercounting by "masking" of bubbles occurred. Additionally, only a simple roller pump was tested as a possible factor in gaseous microemboli delivered to the "patient." It has been demonstrated previously that certain centrifugal pumps function effectively as air microfilters, effectively removing air microbubbles larger than $20 \mu \mathrm{m} .{ }^{5}$

Other components of the $\mathrm{CPB}$ circuit in addition to the arterial pump type are important in generation or elimination of gaseous microemboli. Hollow fiber oxygenators are known to have definite advantages over bubble oxygenators in the avoidance of gaseous microemboli. An inverse relationship exists between gaseous microemboli in the arterial blood and pore size of the bubble oxygenator, with a compromise of oxygenation and carbon dioxide elimination associated with smaller pore sizes. ${ }^{10}$ Therefore, we excluded testing oxygenator type as a possible factor in our experimental series. Additionally, venous reservoir maintenance is associated with the introduction of gaseous microemboli into the $\mathrm{CPB}$ circuit. Running venous reservoirs below certain levels is associated with increased gaseous microemboli. ${ }^{8}$ In the present study, in the test circuits the venous reservoir levels were held constant to compensate for this factor. A lower level was deliberately chosen to emulate a typical clinical scenario.

\section{Limitations of this study}

A possible limitation of our study is the choice of a blood- and albumin-poor solution for our experimental circuits. Although this solution cannot be directly clinically correlated, we chose this fluid to eliminate the effects of blood protein denaturation and coating of the microbubbles, which can be responsible for bubble underestimation by ultrasonic Doppler techniques. ${ }^{14}$ Another possible limitation is that we did not have gas flow through the hollow fiber membrane. Since we were studying gas microemboli from the venous system and gaseous microemboli do not originate from membrane oxygenators, ${ }^{10}$ this should not be of consequence in our experiment.

In summary, assisted venous drainage techniques are associated with an increased risk of gaseous microemboli. In the presence of small constant venous air entrainment, vacuum-augmented venous return is superior to kinetically augmented venous return. When assisted venous drainage techniques are used, a centrifugal arterial pump enhances the removal of signifi- cant gaseous microemboli that may occur despite standard arterial filter precautions.

\section{REFERENCES}

1. Willcox TW, Mitchell SJ, Gorman DF. Venous air in the bypass circuit: a source of arterial line emboli exacerbated by vacuumassisted drainage. Ann Thorac Surg 1999;68:1285-9.

2. Kurusz M, Butler BD. Embolic events and cardiopulmonary bypass. In: Gravlee GP, Davis RP, Utley JR, editors. Cardiopulmonary bypass: principles and practice. Baltimore: Williams \& Wilkins; 1993. p. 267-90.

3. Peters WS, Smith JA, Preovolos A, Rabinov M, Buckland MR, Rosenfeldt FL. Mitral valve replacement via a right mini-thoracotomy in the dog: use of carbon dioxide to reduce intracardiac air. Eur J Cardiothorac Surg 1997;11:1067-73.

4. Semb BK, Pedersen T, Hatteland K, Storstein L, Lilleaasen P. Doppler ultrasound estimation of bubble removal by various arterial line filters during extracorporeal circulation. Scand J Thorac Cardiovasc Surg 1982;16:55-62.

5. Pedersen TK, Karlsen HM. An in-vitro study of six commercially available non occlusive arterial pumps with respect to their handling of free micro gas-bubbles. In: Technical Report by: MTS. Medisinsk Teknisk Sikkerhetskontroll. Ans. Oslo, Norway; 1997.

6. Muth CM, Shank ES. Primary care: gas embolism. N Engl J Med 2000;342:476-82.

7. Pugsley W, Klinger L, Paschalis C, Treasure T, Harrison M, Newman S. The impact of microemboli during cardiopulmonary bypass on neuropsychological functioning. Stroke 1994;25:1393-9.

8. Mitchell SJ, Willcox T, Gorman DF. Bubble generation and venous air filtration by hard-shell venous reservoirs. Perfusion 1997; 12:325-33.

9. De Somer F, Dierickx P, Dujardin D, Verdonck P, Van Nooten G. Can an oxygenator design potentially contribute to air embolism in cardiopulmonary bypass? A novel method for the determination of the air removal capabilities of neonatal membrane oxygenators. Perfusion 1998;13:157-63.

10. Pearson DT. Gas exchange: bubble and membrane oxygenators. Semin Thorac Cardiovasc Surg 1990;2:313-9.

11. Kurusz M, Butler B, Katz J, Conti VR. Air embolism during cardiopulmonary bypass. Perfusion 1995;10:361-91.

12. Toomasian JM, Peters WS, Siegel LC, Stevens JH. Extracorporeal circulation for port-access cardiac surgery. Perfusion 1997;12:83-91.

13. Groh MA, Fallen DM. Alteration of the traditional extracorporeal bypass circuit to accommodate port-access minimally invasive cardiac procedures using endovascular based cardiopulmonary bypass. Artif Organs 1998;22:775-80.

14. Butler BD. Biophysical aspects of gas bubbles in blood. Med Instrum 1985;19:59-62.

\section{Discussion}

Dr Verdi DiSesa (Chicago, Ill). I have a question about the first conclusion in your summary slide, which indicates an increased risk of gaseous microemboli when assisted circulation is used. To be fair, would it not be necessary to do the 
same experiments in a gravity sump system, including inserting a 25-gauge needle, to prove that?

Dr LaPietra. Even though the results of venous air handling with conventional gravity siphon drainage are not presented, we have performed this part of the experiment in our laboratory to verify the results that others have recently published. Using a similar model as in the presented data, we tested the venous air handling ability of gravity siphon drainage. We have found that with an open 25-gauge needle inserted into the venous line and a 40-cm height differential between our simulated patient and the venous reservoir, less air was introduced into the circuit, when compared with the augmented systems. As mentioned earlier, we did not include this part of the experiment in our paper since others have published these results.

Dr Ludwig von Segesser (Lausanne, Switzerland). I think it is important to state that it is not necessary to introduce air into the venous line to have air in the system. It is enough to have a clamp on the arterial side and maintain vacuum on the venous side. The air will come through the oxygenator.

Dr LaPietra. Thank you for your comments. Your point is well taken, Dr von Segesser. Although this is absolutely true, it is not a situation that would happen in a normal clinical setting. Most of the components of the CPB circuit have been evaluated in their air-handling abilities with special emphasis on the oxygenator type and arterial pump type. Now we are trying to build on some of these findings to evaluate how these components behave under augmented assisted venous drainage.

Dr Peter P. McKeown (Asheville, $N C$ ). I want to compliment you on extending our understanding of CPB as we also look at off-pump procedures. We have actually done away with cardiotomy suction for most of the standard coronary bypass operations. I guess that Shukri Khuri, Westaby, and others have believed that the blood-air interface is a real problem. I wanted to ask about that. Did you actually just use the cell salvage system? Also, did you use heparin-bonded circuits or heparin-bonded membranes?

Dr LaPietra. Thank you, Dr McKeown. All of the circuits that were constructed in this experiment consisted of heparinbonded tubing and membranes, such as we would normally use in our clinical practice. However, we did not use any blood components in our experiment, so the importance of heparin coating was minimized. We did not use cardiotomy suction and a cell salvage device in this experiment.

Dr Craig R. Smith (New York, NY). Do you have an opinion as to what constitutes a clinically relevant number or size of bubbles?

Dr LaPietra. The literature seems to favor microbubbles greater than $100 \mu \mathrm{m}$ as being clinically significant. However, we have no clinical data to verify this number. In our experiment, some of our designs allowed for bubbles 50 to $100 \mu \mathrm{m}$ to pass to the patient side of the circuit. The significance of these sizes is not yet clear. I believe that some neurocognitive studies with respect to bubble sizes need to be evaluated clinically to determine whether there actually is a critical size.

Dr Edward D. Verrier (Seattle, Wash). Clinically, has air caused any difficulties? Vacuum-assisted systems have been used for a long time at the Cleveland Clinic, but I do not know whether any problems have been encountered. Our perfusionists at the University of Washington have not noticed any problems with centrifugal pumps, and we are sensitive to difficulties caused by air in the system. We have not used roller pumps. I am curious about whether this is a clinical problem or just a warning for us to be very careful in setting up our systems.

Dr LaPietra. From our perspective, it is basically a warning on how the circuit is constructed. In our practice, we are currently using vacuum-augmented venous return with an arterial centrifugal pump, and we have not experienced any problems either. However, on the basis of these findings, we believe the use of an arterial roller pump with the addition of vacuum-assisted venous drainage needs to be cautioned. Obviously, the perfusionist plays a major role in circuit design and setup. However, it behooves us as surgeons to know the components and types of circuits being used clinically to prevent any neurologic complications that could occur during CPB.

Dr Verrier. Would you recommend a regulatory warning with regard to combining this vacuum drainage with the roller pump?

Dr LaPietra. No, we do not think that a regulatory warning is required. However, we do think that clinical neurocognitive studies should be performed to evaluate the different augmented venous drainage techniques being used. 